\title{
Relationship between Eastern Cooperative Oncology Group performance status (ECOG-PS) and actigraphy-derived activity parameters in a heterogeneous group of advanced cancer patients \\ Dr Shuchita Dhwiren Patel, ${ }^{1,2}$, Dr Andrew Davies ${ }^{1,2}$, Prof Derk-Jan Dijk ${ }^{2}$ \\ ${ }^{1}$ Royal Surrey County Hospital, ${ }^{2}$ University of Surrey, Guildford, U.K.
}

\section{INTRODUCTION}

Performance status has been shown to be a reliable predictor of overall survival ${ }^{1}$. However, discrepancies often occur between different assessors (as this is a subjective assessment) ${ }^{2}$. Actigraphy may be a better, objective method for prognostication. The actigraphy-derived dichotomy index $(1<0)$, a marker of the daily rest-activity rhythm, is a measure that compares the amount of activity at night-time (major sleep period) to activity in the daytime (major wake period) ${ }^{3}$. The $\mathrm{K}<\mathrm{O}$ has been reported to be of prognostic value in the metastatic colorectal cancer population ${ }^{4}$. However, the association between the ECOG-PS and $1<0$ has not been fully explored.

The objectives of the study were to assess the relationship between ECOG-PS scores and the $1<0$ and other actigraphy-derived activity parameters such as $\mathrm{r24}$, mean daytime activity, and mean 24-hour activity, and to compare ECOG-PS scores assessed by the palliative care physician and patients.

\section{METHODS}

50 patients with advanced cancer and an estimated prognosis of $<1$ year were recruited as part of a feasibility study. Patients and the palliative care physician independently assessed the patient's ECOG-PS at baseline and after 7 days. Participants were instructed to wear an Actiwatch Spectrum Plus ${ }^{\circledR}$ for seven consecutive 24-hour periods on their nondominant arm, and to concurrently complete a sleep diary. Spearman's rank correlation coefficient was used to assess the associations between physician-assessed ECOG-PS scores and the actigraphy-derived parameters.

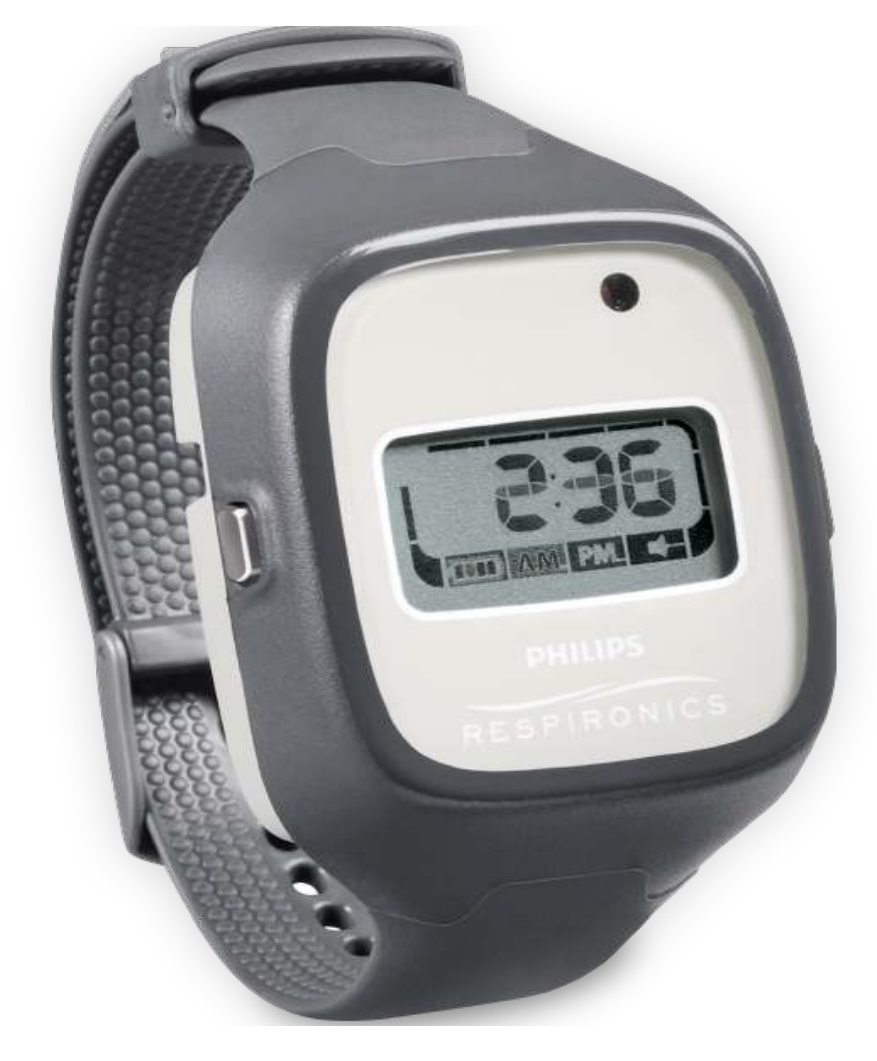

\section{RESULTS}

ECOG-PS assessments were completed in 49 patients. On Day 8, there was moderate agreement between the palliative care physician and the patient assessments of their ECOG-PS, with a Kendall's correlation of 0.70 $(p<0.001)$ and kappa value of $0.47(p<0.001)(95 \% \mathrm{Cl} 0.66,0.28)$.

44 patients wore the Actiwatch for at least 72 hours. A moderate negative correlation was observed between physician-assessed ECOG-PS and the dichotomy index $(1<0)(r=-0.57 ; p<0.0005)$. A low negative correlation was observed with ECOG-PS and $r 24(r=-0.34 ; p=0.026)$, as well as, ECOG-PS and mean daytime activity $(r=-0.35 ; p=0.021)$. There was no correlation between physician-assessed ECOG-PS and mean 24-hour activity level $(r=-0.24 ; p=0.115)$.
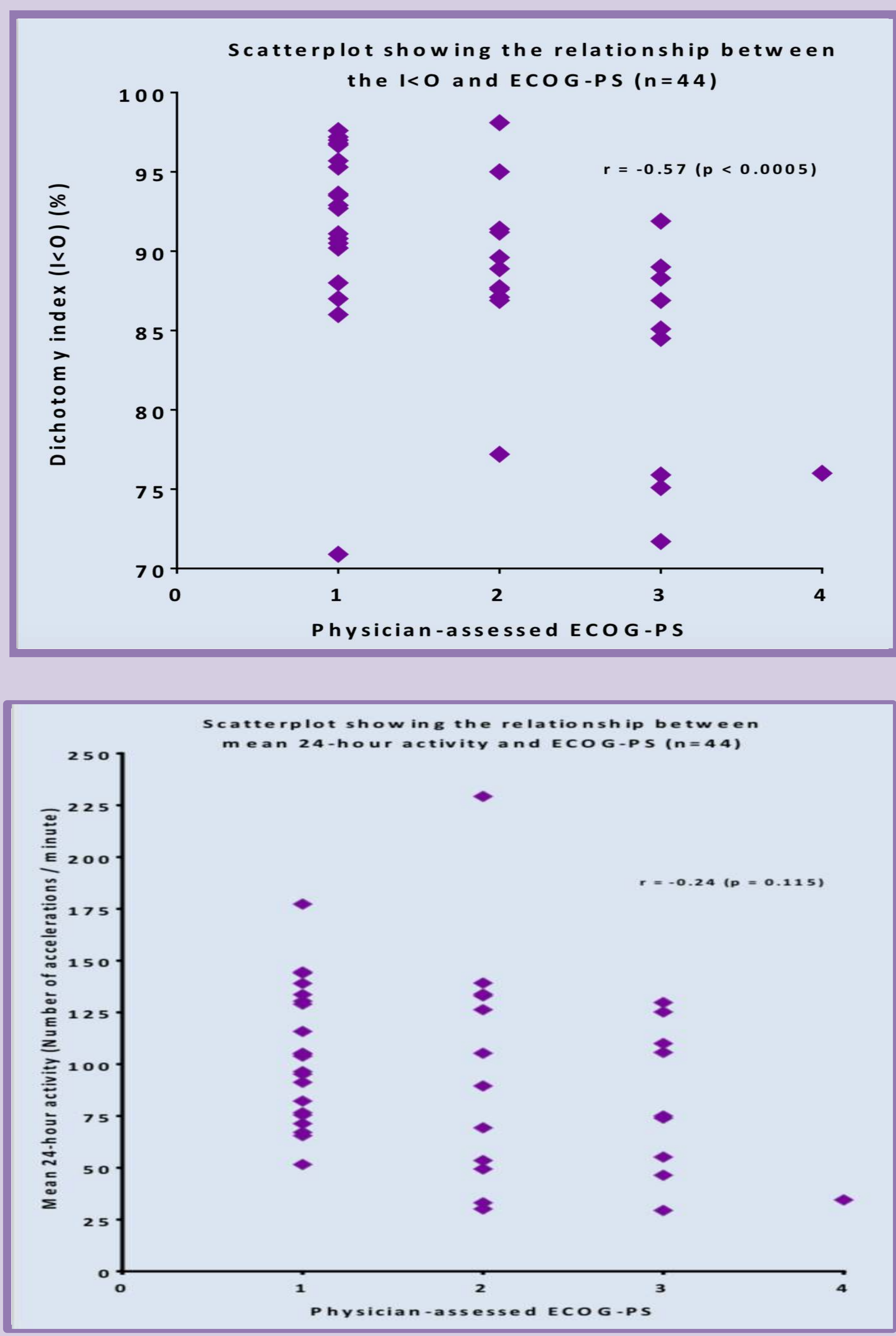

\section{CONCLUSION}

Mean total activity counts derived from actigraphy do not correlate with ECOG-PS, and so would not be expected to be good prognostic indicators. However, the dichotomy index $(1<0), \mathrm{r} 24$ and mean day-time activity are correlated with ECOG-PS, and may be good (objective) prognostic indicators in patients with advanced cancer.

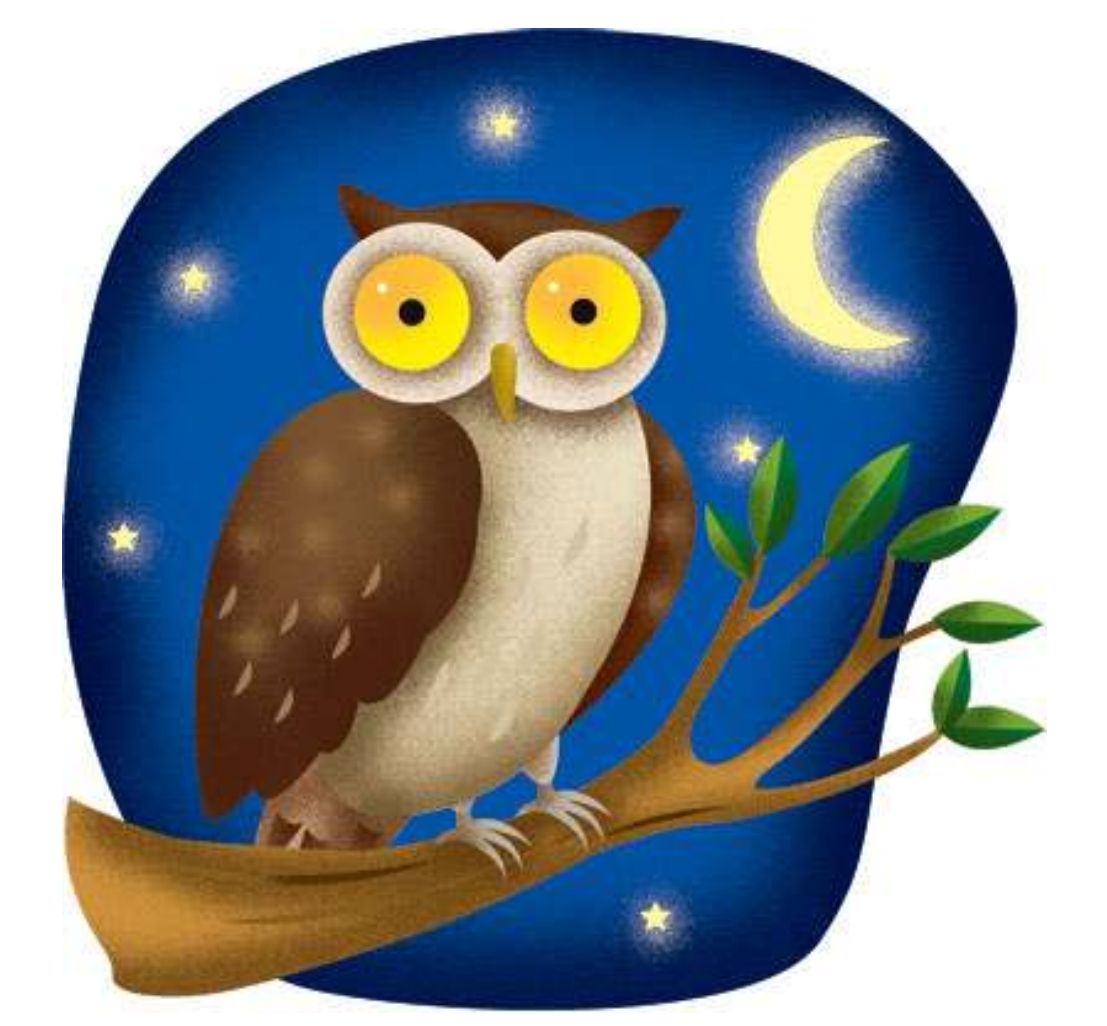

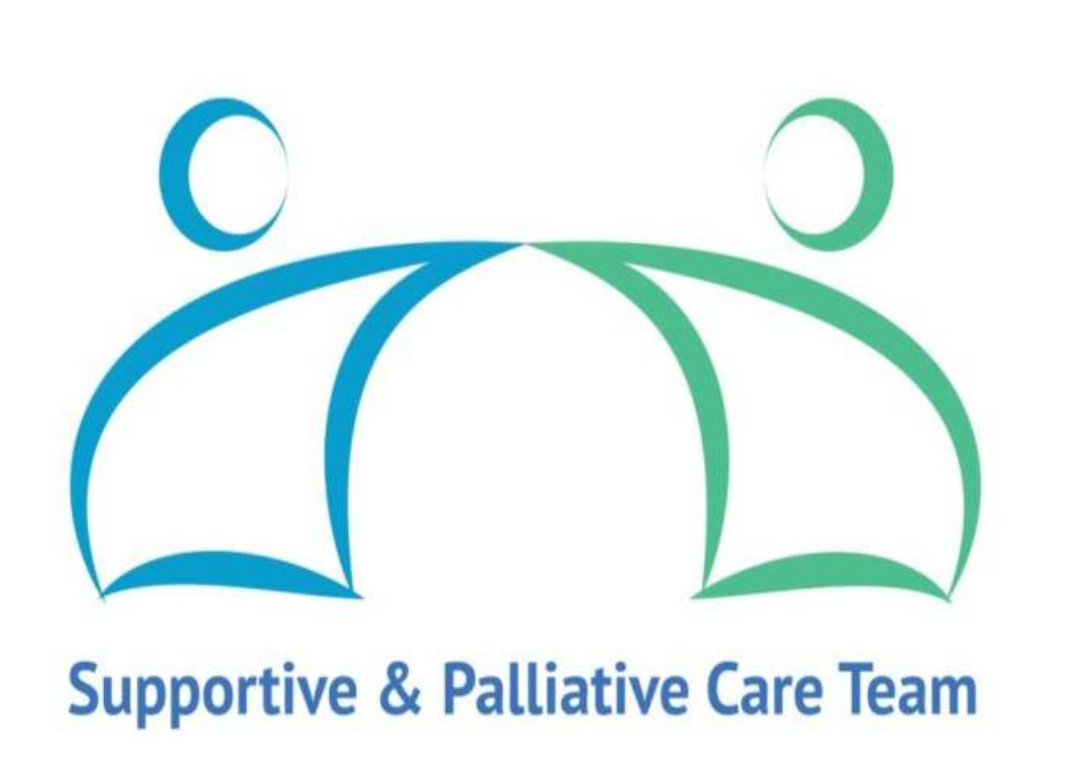

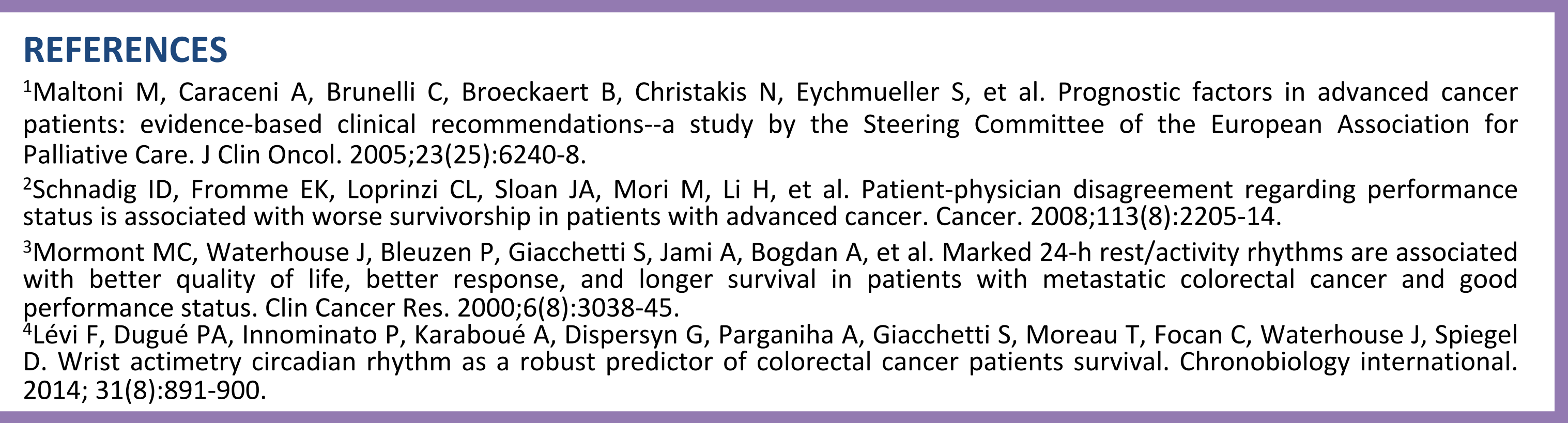

